IJASC13-1-5

\title{
A Study on Mobile Target Estimation Resolution using Effects of Model Errors and Sensitivity Analysis
}

\author{
Kwan Hyeong Lee* \\ *Dept. Communcation Engineering Daejin University, \\ 1007 Hogukro, Phocheon, Gyeonggi, Korea. \\ khlee@daejin.ac.kr
}

\begin{abstract}
The antenna pattern in this case has a main beam pointed in the desired signal direction, and has a null in the direction of the interference. The conventional antenna pattern concepts of beam width, side lobes, and main beams are not used, as the antenna weights are designed to achieve a set performance criterion such as maximization of the output SNR.A new direction of arrival estimation method using effects of model errors and sensitivity analysis is proposed. Two subspaces are used to form a signal space whose phase shift between the reference signal and its effects of model error signal. Through simulation, the performance showed that the proposed method leads to increased resolution and improved accuracy of DOA estimation relative to those achieved with existing method. Since a desired signal is obtained after interference rejection through correction effects of model error, the effect of channel interference on the estimation is significantly reduced.
\end{abstract}

Keywords: Model error, Sensitivity, Estimation, Resolution, MUSIC

\section{INTRODUCTION}

This is multi dimension estimation problem, and thus the necessary signal processing algorithms are complex [1]. The complexity of these estimation algorithm is further magnified when array model errors such as the inequality of sensor channel gain and phase characteristics, sensor location errors, and the presence of inter sensor mutual coupling are included in the numerical model[2]. One way of reducing the complexity of the estimation algorithm is to characterize the array model more accurately by estimating the net effect of the various errors and using this real array model in the parameter estimation [3]. This requires signal sources with known parameters covering the utilized array manifold. The adaptive array antennas are used for a phased array when the weighting on each element is applied in a dynamic fashion. The amount of weighting on each channel is not fixed at the time of the array design, but

Manuscript received:Jan. 11, 2013 / revised :Apr. 13, 2013

Corresponding Author: khlee@daejin.ac.kr

Tel:+82-31-539-1925,Fax: +82-31-539-1925

Dept. of Communication Engineering Daejin University, Korea rather decided by the system at the time of processing the signals to meet required objectives. In other words, the array pattern adapts to the situation and the adaptive processing is under control of the system. For example, consider the situation of a communication system operating the inter presence of a directional interference operating at the carrier frequency used by the desired signal, and the performance measure is to maximize the output SNR. As discussed previously, the output SNR is maximized by canceling the directional interference using optimal antennas. The antenna pattern in this case has a main beam pointed in the desired signed direction, and the performance measure is to maximize by canceling the directional interference using optimal antennas. The antenna pattern in this case has a main beam pointed in the desired signal direction, and has a null in the direction of the interference. Assume that the interference is not stationary but moving slowly.

If optimal performance is to be maintained, the antenna pattern needs to adjust so that the null position remains in the moving interference direction. A system using adaptive antennas adjusts the weighting on each channel with and 
aim to achieve such a pattern. The conventional antenna pattern concepts of beam width, side lobes, and main beams are not used, as the antenna weights are designed to achieve a set performance criterion such as maximization of the output SNR. On the other had in conventional phase array design these characteristics are specified at the time of design.

In the proposed DOA estimation method, the target DOA is estimated from the phase shift introduced in the target signal by effects model error, which is a function of the target DOA. Since the phase shift is estimated after effects of model error, all signals and interference other than the target one can be efficiently rejected before DOA estimation. Thus their interference on the DOA estimation is reduced. In this way, the estimation resolution and accuracy of the proposed method are better than those for existing method.

\section{DOA Antenna Signal Model}

Consider an antenna composed of $M$ antenna sensors arbitrarily located in spatial and assume that a signal impinges on the array. Then the output of $m^{\text {th }}$ the sensor can be written as follow [4-5]

$\mathrm{X}_{m}(\mathrm{t})=\sum_{n=1}^{p} \alpha_{m} s_{n}\left(t-\tau_{m n}\right)+n_{m}(t)$

Where $\mathrm{s}_{n}(t)(n=1,2, \cdots, p)$ is the receive signal, $\alpha_{m}$ and $\varphi_{m}$ are the gain and phase delay associated with the $m^{\text {th }}$ sensor , $\tau_{m n}$ is the delay relative to a reference point associated with the signal propagation from the $n^{\text {th }}$ source to the $m^{\text {th }}$ sensor. The array output can be expressed as [7]

$X(\mathrm{t})=\mathrm{C} \Lambda \mathrm{A}(\theta) S(t)+N(t)$

where $\mathrm{X}(\mathrm{t})$ is the received signal vector, $\mathrm{C}$ is the mutual coupling matrix, $\Lambda$ is complex diagonal matrix, $S(t)$ is the signal vector, $\mathrm{A}(\theta)$ is array manifold matrix, $a\left(\theta_{m}\right)$ is steering vector corresponding to the $m^{\text {th }}$ source at direction $\theta_{m}$, and $\mathrm{N}(\mathrm{t})$ is additive white noise.In subspace DOA estimation methods, the array output covariance matrixR can be partitioned into two subspace, the signal subspace and the noise subspace, using eigenvalue decomposition, such that

$R=\mathrm{E}_{s} \gamma_{s} E_{s}^{H}+\sigma^{2} E_{n} E_{n}^{H}$

Where $\mathrm{R}$ is signal covariance matrix, ()$^{H}$ is Hermitean. $\mathrm{E}_{s}$ and $\mathrm{E}_{n}$ are signal subspace and noise subspace respectively. The array manifold will affect the orthogonality between the two subspaces. We refer to the different between the ideal and real array parameters. The spatial spectrum of MUSIC and DOA estimation can be written as follow

$$
\operatorname{SE}(\theta, \mu)=\frac{1}{A(\theta) E_{n}^{H}(\theta) E_{n}(\theta) A^{H}(\theta)}
$$

\section{Simulation}

General methods used ML and MUSIC method. Consider a uniform linear array with 9 sensors, each sensor separated by half wavelength. Fig.2 shows the Root Mean Square Error(RMSE) of the DOA estimation of a signal arriving using the ML, MUSIC and proposed method for different SNR. The SNR is varied from $0 \mathrm{~dB}$ to $25 \mathrm{~dB}$. In Fig.1, ML method showed $10^{-1}$ in the case of $\mathrm{SNR}=10 \mathrm{~dB}$, MUSIC method shows $10^{-1.6}$ in the case of $\mathrm{SNR}=10 \mathrm{~dB}$, and proposal method shows $10^{-1.8}$ in the case of $\mathrm{SNR}=10 \mathrm{~dB}$. Proposal method improved $8 \mathrm{~dB}$ than ML method in this paper. Proposal method improved 2dB than MUSIC method. ML method shows SNR $10 \mathrm{~dB}$ in the case of MMSE $10^{-1}$. MUSIC method shows SNR 6dB in the case of MMSE $10^{-1}$. Proposal method shows SNR $4 \mathrm{~dB}$ in the case of MMSE $10^{-1}$.

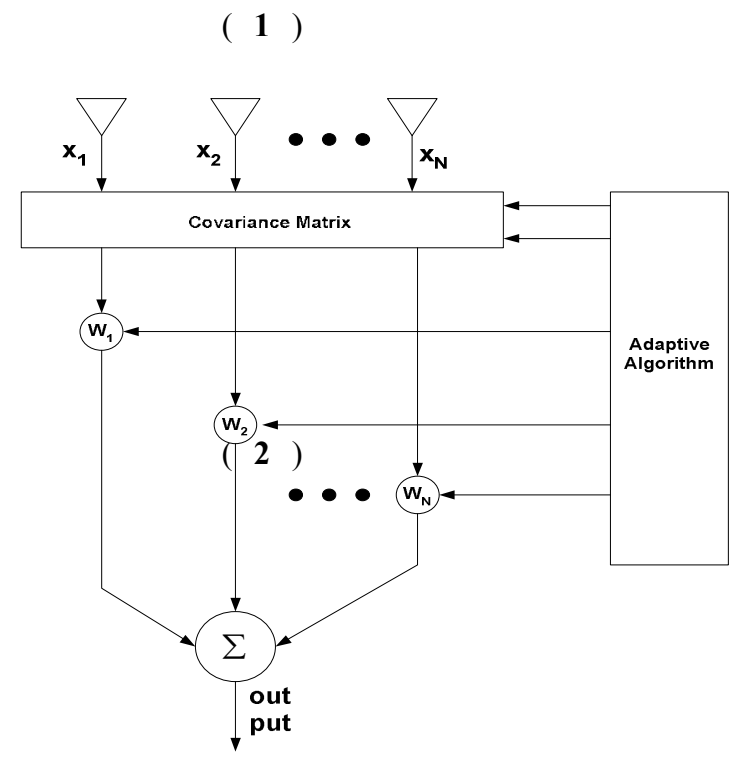

Fig. 1. Adaptive Array Antenna system 


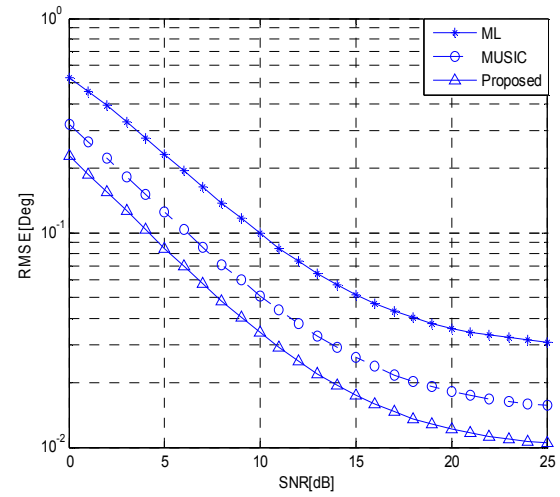

Fig. 2. RMSE proposed method and existing method

\section{CONCLUSION}

A new DOA estimation method based upon effects of model errors has been proposed. In the new method, two subspaces are used to obtain an optimum estimation of the desired signal whose phase relative to the model error signal is a function of the target DOA. Through simulation, the performance showed that the proposed method leads to increased resolution and improved accuracy of DOA estimation relative to those achieved with existing method.

\section{REFERENCES}

[1] EDMOND NICOLAU.: Adaptive Arrays, ELSEVIER Press, NewYork(1983)

[2] J.E.Hudson.: Adatpive Array Principles, IEE Press, London (1989)

[3] B.Friedlander.: A Sensitivity Anslysis of the MUSIC Algorithm. IEEE Transaction on Acoustics, Spech, and Signal Processing Vo.30, No.10, pp.31--34, IEEE Press, NewYork(1990)

[4] A.J.weiss and B.Friedlander.: Eigen structure Methods for Direction Findng with Sensor Gain and Phase Uncertainites. IEEE Transaction on Signal Processing, Vo.9, No.3, pp.272--300, IEEE Press, NewYork(1990)

[5] E.M Friel and K.M.Pasala.: Wideband Bearing Estimation with Compensation for Mutual Coupling Effects. IEEE Antenna and Propagation Symposium(AP-SURSI94), Vo.3, pp.1556--1559, IEEE Press, NewYork(1994)

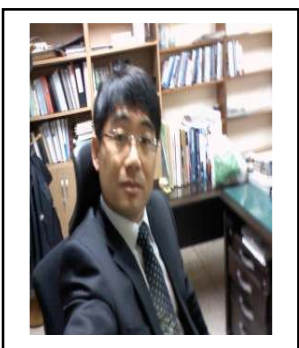

Kwan Hyeong Lee

Receivedph.D degree in Electronics Engineering from cheongju university, cheongju, korea, in 2004 respectively. From 1998 to 2004, He was work an associate professor, Dep.

Information\&communicationGang neungyeongdong college. From 2005 to 2007 , He was work at full time lecture in cheongju university. From 2007 to 2010, He was work at Agency for defense development. Currently, Heisworking an associate professor in daejin university. Heis interested the field that like to wireless communication and sensor network. 